\title{
CT stress perfusion imaging for detection of haemodynamically relevant coronary stenosis as defined by FFR
}

\author{
Martin Greif, ${ }^{1}$ Franz von Ziegler, ${ }^{1}$ Fabian Bamberg, ${ }_{1}{ }^{2}$ Janine Tittus, ${ }^{1}$ Florian Schwarz, ${ }^{2}$ \\ Melvin D'Anastasi, ${ }^{2}$ Roy P Marcus, ${ }^{2}$ Jan Schenzle, ${ }^{1}$ Christoph Becker, ${ }^{2}$ \\ Konstantin Nikolaou, ${ }^{2}$ Alexander Becker ${ }^{1}$
}

'Department of Cardiology, Klinikum Grosshadern, University Hospital of Munich, Munich, Bayern, Germany ${ }^{2}$ Department of Radiology, Klinikum Grosshadern, University Hospital of Munich, Munich, Bayern, Germany

\section{Correspondence to} Dr Martin Greif, Department of Cardiology, Klinikum Grosshadern, University Hospital of Munich, Marchioninistrasse 15, Munich 81377, Bayern, Germany; martin.greif@med.unimuenchen.de

Received 12 February 2013 Revised 16 April 2013 Accepted 17 April 2013 Published Online First 14 May 2013
To cite: Greif $M$, von Ziegler F, Bamberg F, et al. Heart 2013;99:1004-1011.

\begin{abstract}
Objectives To evaluate the diagnostic accuracy (DA) of CT-myocardial perfusion imaging (CT-MPI) and a combined approach with CT angiography (CTA) for the detection of haemodynamically relevant coronary stenoses in patients with both suspected and known coronary artery disease.
\end{abstract}

Design Prospective, non-randomised, diagnostic study.

Setting Academic hospital-based study.

Patients 65 patients (42 men age 70.4 \pm ) with typical or atypical chest pain.

Interventions CTA and CT-MPI with adenosine stress using a fast dual-source CT system. At subsequent invasive angiography, FFR measurement was performed in coronary arteries to define haemodynamic relevance of stenosis.

Main outcome measures We tried to correlate haemodynamically relevant stenosis (FFR $<0.80)$ to a reduced myocardial blood flow (MBF) as assessed by CT$\mathrm{MPI}$ and determined the DA of CT-MPI for the detection of haemodynamically relevant stenosis.

Results Sensitivity and negative predictive value (NPV) of CTA alone were very high ( $100 \%$ respectively) for ruling out haemodynamically significant stenoses, specificity, Positive predictive value (PPV) and DA were low $(43.8,67.3$ and $72 \%$, respectively). CT-MPI showed a significant increase in specificity, PPV and DA for the detection of haemodynamically relevant stenoses (65.6, 74.4 and $81.5 \%$, respectively) with persisting high sensitivity and NPV for ruling out haemodynamically relevant stenoses ( $97 \%$ and $95.5 \%$ respectively). The combination of CTA and CT-MPI showed no further increase in detection of haemodynamically significant stenosis compared with CT-MPI alone.

Conclusions Our data suggest that CT-MPI permits the detection of haemodynamically relevant coronary artery stenoses with a moderate DA. CT may, therefore, allow the simultaneous assessment of both coronary morphology and function.

\section{INTRODUCTION}

Cardiac CT has established itself as a valuable tool for physicians. The coronary artery calcium score is an important and worthwhile parameter for cardiovascular risk stratification. By using CT angiography (CTA) in patients with suspected coronary artery disease (CAD), significant coronary artery stenoses can be ruled out non-invasively and with a high diagnostic accuracy (DA). Coronary artery stenoses can be visualised by CTA, and the grade of stenosis can be determined. However, the haemodynamic significance of any detected stenosis could so far not be evaluated by CT because the acquired information was strictly morphologic in nature. The haemodynamic significance of a coronary artery stenosis had to be assessed by other imaging modalities, such as single-photon emission CT (SPECT), or stress myocardial perfusion MRI. These modalities have been shown to deliver reliable information about myocardial perfusion defects at rest and under stress conditions, however, they do not provide information about the morphology of the coronary vessel and the causal stenosis. So far, morphologic and functional information could only be obtained in the same examination invasively by coronary angiography and fractional flow reserve measurement.

From a clinical point of view, it is crucial to identify the haemodynamically significant stenosis, because only the treatment of the latter results in reduced mortality and myocardial infarction, as shown by the FAME ${ }^{1}$ and the COURAGE study. ${ }^{2}$

Over the last few years, a number of initial studies have reported that the detection of myocardial perfusion defects, at rest or even stress-induced defect, ${ }^{3-6}$ is feasible if using CT. Two recent small patient series could also show that detection of myocardial perfusion defects at rest or under stress conditions is viable. ${ }^{7}{ }^{8}$ It was the aim of this study to investigate the feasibility of myocardial perfusion imaging (MPI) to detect haemodynamically significant coronary artery stenoses, as defined by FFR, in a larger series of patients.

\section{METHODS \\ Patients}

Sixty-seven patients (43 men; age 70.6 49 ) presenting at our institution with typical or atypical chest pain (74.3\% with known CAD, 49\% with a history of stent implantation, $9.4 \%$ with a history of bypass surgery) or suspected CAD were included. Patients with unstable angina, prior myocardial infarction, atrial fibrillation, serum creatinine levels greater than $1.5 \mathrm{mg} / \mathrm{dl}$, atrioventricular block greater than type I, or patients younger than 50 years, were excluded. ß-adrenergic blocking agents were discontinued at least 1 day before CT-MPI, no coffee or tea was allowed before the examination. 
All patients signed written informed consent. The study was approved by the institutional review board and the Federal Radiation Safety Council (Bundesamt für Strahlenschutz).

Further patients characteristic are given in table 1.

\section{CT angiography and CT stress myocardial perfusion}

Images were generated by using a fast dual-source CT system (Somatom Definition Flash; Siemens Healthcare, Forchheim, Germany) with a collimation of $64 \times 2 \times 0.6 \mathrm{~mm}$ and flying focus, resulting in $2 \times 128$ sections. Two intravenous lines were placed in both antecubital veins for administration of contrast agent and adenosine. After scout images and administration of a test bolus (injected at $5 \mathrm{ml} / \mathrm{s}$ ) of contrast agent (Ultravist 370; Bayer Schering Pharma), standard prospective CTA was performed. Because of possible interference with adenosine efficacy, no $B$-adrenergic blocking agent was applied. CTA was triggered at $200 \mathrm{~ms}$ in the craniocaudal direction. Further standard scan parameters were as follows: $2 \times 100 \mathrm{kVp}$ tube voltage $(120 \mathrm{kVp}$ in patients with a BMI $>30 \mathrm{~kg} / \mathrm{m}^{2}$ ), $320 \mathrm{mAs}$ per rotation, and 0.28 -s gantry rotation time. Evaluation of images and determination of significant coronary artery stenoses (>50\%) was performed by two independent experienced investigators (FS and FvZ) using a modified 17-segment model of the coronary artery tree. ${ }^{9}$ Both were blinded to perfusion results as well as coronary angiography results. If a significant stenosis in a segment could not be ruled out, a stenosis of $>50 \%$ in this segment was assumed.

After 3 min of continuous adenosine administration (Adrekar; Sanofi, Munich, Germany) at an infusion rate of $0.14 \mathrm{mg} / \mathrm{kg} /$ min, MPI was started.

Data acquisition was performed for $30 \mathrm{~s}$ with both tubes set at $100 \mathrm{kV}$, a gantry rotation time of $0.28 \mathrm{~s}$, and a total tube current of $300 \mathrm{mAs}$ per rotation. In the electrocardiographically triggered mode the table was shifting between two positions ('shuttle mode,' table acceleration time $300 \mathrm{~mm} / \mathrm{s}^{2}$ ). With a defined detector width of $38 \mathrm{~mm}$, and a 10\% overlap between both imaging positions, the coverage of the acquisition was $73 \mathrm{~mm}$. A total of 14-15 datasets were acquired. Overall, $50 \mathrm{ml}$ of iodinated contrast agent (Ultravist 370; Bayer Scherimg Pharma; flow rate $5 \mathrm{ml} / \mathrm{s}$.) was needed to generate the images. Two independent experienced readers (FB and $\mathrm{AB}$ ) performed image analysis using a dedicated software tool on our workstation (Leonardo; Siemens Medical Solutions, Erlangen, Germany). These readers were blinded to CT angiography results.

Perfusion images were reconstructed with a $3 \mathrm{~mm}$ section width every $2 \mathrm{~mm}$ by using a smooth convolution kernel (B30),

\section{Table 1 Baseline characteristics}

\begin{tabular}{ll}
\hline $\mathrm{N}$ & All \\
\hline Age & 65 \\
Male & $70.4 \pm 9$ \\
Known CAD & 42 \\
History of stent implantation\% & 74.3 \\
History of CABG\% & 49.0 \\
Hypercholesterolemia\% & 9.4 \\
Hypertension\% & 47.6 \\
Smoking\% & 67.3 \\
Family history of CAD & 25.4 \\
Diabetes \% & 33.4 \\
\hline CAD, coronary artery disease. & 17.9 \\
\hline
\end{tabular}

and then processed on a standard workstation (Syngo VPN; Siemens Healthcare, Forchheim, Germany). Myocardial blood flow (MBF) and mean attenuation in Hounsfield units were determined for each of the 16 myocardial segments, excluding the apical segment. A $1 \mathrm{~mm}$ subendocardial zone directly adjacent to the contrast material-filled left ventricle, and a $1 \mathrm{~mm}$ subepicardial zone were excluded from analysis. A region of interest of $2.5 \mathrm{~cm}^{2}$ was manually placed in a representative area of each myocardial segment. To estimate MBF, a dedicated parametric deconvolution technique, which was based on a twocompartment model of intravascular and extravascular space, was used to fit the time-attenuation curves. ${ }^{10}$ The precision of the fit is increased significantly by double sampling of the arterial input function (AIF). The input function is sampled from regions of interest (ROIs) placed in the descending aorta with every table position and combined into one AIF that has twice the sampling rate of the tissue time-attenuation curve (TAC). The algorithm then determines the maximum slope from the fit model curve for every voxel, and calculates MBF according to the following relationship: $\mathrm{MBF}=(\mathrm{MaxSlope}($ TissueTAC $))$ (Maximum(AIF)). ${ }^{11}$

The intraclass correlation coefficient was used to determine the agreement of measurement of MBF between two observers. A paired t test and repeated-measures analysis of variance was used to compare MBF between segments, which were and were not associated with a haemodynamically significant coronary artery stenosis and according to CT stenosis category.

To determine whether the observed difference between territories was independent of heart rate during the scan, age, sex and body mass index, we fitted linear regression models by using the MBF as the predictor of interest. The MBF cut point was derived by maximisation of the $\mathrm{C}$ statistic by using logistic regression modelling. ${ }^{12}$ The asymptomatic 95\% CIs for the C statistic were estimated by using a non-parametric approach, which is closely related to the jack-knife technique proposed by DeLong et al. ${ }^{13}$

\section{Conventional coronary angiography}

All patients underwent coronary angiography 1-3 days after CT-MPI. Coronary angiography was performed as a standard procedure via femoral access using a $6 \mathrm{~F}$ guiding catheter in our catheter laboratory (Siemens Medical Solutions, Forchheim, Germany). Each coronary segment was visually assessed for degree of luminal stenosis by two independent experienced invasive cardiologists ( JT and MG) who were blinded to the CT findings. If a 50-85\% luminal stenosis was detected, a fractional flow reserve measurement was performed.

\section{FFR measuring}

Using a sensor tipped 0.014-inch guidewire (Prime Wire Prestige; Volcano, Japan) coronary artery stenosis suspected to be haemodynamically relevant (lumen narrowing between 50\% and $85 \%$ ), were evaluated as follows. After placement of the pressure wire distal to the target lesion, a continuous intravenous infusion of adenosine at an infusion rate of $0.14 \mathrm{mg}$ per kilogram of body weight per minute, similar to infusion rate for MPI-CT, was started. FFR was calculated by dividing the mean coronary pressure, measured by the pressure wire distal to the stenosis by the mean proximal pressure measured by the guiding catheter. If within $3 \mathrm{~min}$, the FFR was 0.80 or less, the stenosis was considered haemodynamically relevant. Stenosis greater than $90 \%$ clearly heamodynamically significant or totally occluded vessels were considered functionally significant without performing FFR measurement. This procedure was 
repeated for all coronary arteries having a stenosis of $50-85 \%$ by angiographic evaluation.

\section{Statistical analysis}

Statistical analyses were performed using the SPSS software package (V.18.0, SPSS, Chicago, Illinois). All values are expressed as mean score \pm SD exceptions were indicated. Values were compared between groups with a two-tailed unpaired Student $\mathrm{t}$ test for continuous data, whereas, categorical data were compared using an $\times 2$ or Fisher exact test. A $p<0.05$ was considered as significant. DA was compared using the McNemar Test. All values illustrated in the figures are given as mean \pm SD.

\section{RESULTS}

Of 67 enrolled patients, 65 completed the CT scan. Two patients had to be excluded from further analysis due to technical failure. The two technical failures occurred in the first 20 patients, following which no more technical failures occurred. Thus, 65 patients formed our study cohort.

Average scan duration was $34.7 \pm 6.3 \mathrm{~min}$. Mean effective radiation exposure was $2.9 \pm 0.9 \mathrm{mSv}$ for CTA, and 9.7 $\pm 2.2 \mathrm{mSv}$ for CT myocardial perfusion. $80 \mathrm{ml}$ of contrast agent were used for CTA and $50 \mathrm{ml}$ of contrast agent for CT -MPI. Heart rate at baseline was $67 \pm 23 \mathrm{bpm}$. This showed a significant increase after adenosine administration up to $81 \pm 14 \mathrm{bpm}(\mathrm{p}<0.001)$. Complete coverage of the myocardium could be achieved in 49 patients (77.7\%). In 11 patients $(17.4 \%)$ the inferior territories and in three patients $(4.7 \%)$ the anterior territories of the myocardium were only partially covered. Seven studies (11.1\%) showed reduced image quality, mainly to motion artefacts, and required secondary postprocessing; 31 not evaluable segments were rated as segments without perfusion defects. In case of haemodynamically relevant stenosis in FFR, these patients would have been considered as false negative. No examination was completely excluded from analysis.

\section{MBF cut-off}

Myocardial segments pertaining to haemodynamically significant coronary artery lesions had significantly lower mean MBF $(78.7 \mathrm{ml} / 100 \mathrm{ml} / \mathrm{min} \pm 26.1$ vs $122.7 \pm 34 \mathrm{ml} / 100 \mathrm{ml} / \mathrm{min}$ ), $(\mathrm{p}<0.001$ for both). The predicted difference in MBF between ischaemic and non-ischaemic myocardial segments persisted after adjusting for age, sex, body mass index and difference in heart rate $(B, 36.2 \mathrm{ml} / 100 \mathrm{ml} / \mathrm{min} ; 95 \% \mathrm{CI}$ : $26.8,43.1$; $\mathrm{p}<0.001)$. The best cut-off of $\mathrm{MBF}$ for the differentiation between haemodynamically significant and non-significant coronary artery stenosis was $75 \mathrm{ml} / 100 \mathrm{ml} / \mathrm{min}$ (c statistic, 0.712 ; $\mathrm{p}<0.001)$.

\section{Conventional angiography}

All 65 patients included in the study underwent conventional angiography. In 10 patients, no coronary stenosis $\geq 50 \%$ was found; 55 patients showed at least one stenosis $\geq 50 \%$; 33 of these patients had a haemodynamically relevant stenosis detected by FFR, and underwent stent implantation or coronary artery bypass surgery.

\section{CTA for the detection of haemodynamically relevant coronary stenoses}

All 65 patients underwent CTA. CTA detected all haemodynamically relevant coronary stenoses resulting in a sensitivity of $100 \%$. The specificity of CTA for the detection of a haemodynamically significant coronary stenosis was low, with $43.8 \%$. A positive predictive value (PPV) of $67.3 \%$ was calculated. Even though CTA had many false positive findings, the negative predictive value (NPV) was 100\%. All seven patients with exclusion of a significant coronary artery stenosis in CTA had no haemodynamically relevant stenosis revealed by coronary angiography.

In 58 patients, CTA revealed at least one stenosis $\geq 50 \%$, in one of their coronary artery vessels. Of these 58 patients, only 33 patients had a haemodynamically significant stenosis detected by FFR, 25 had no haemodynamically significant stenosis. Overall sensitivity, specificity, negative and PPV and DA of CTA for the presence of a haemodynamically significant stenosis were 100, 43.8, 100, 67.3, and 72\%, respectively. These findings remained unaffected with a high sensitivity, a high NPV, a low specificity, and a low PPV in a vessel-based analysis. There was no significant difference in DA between the single coronary arteries (see table 2).

\section{CT-MPI for the detection of haemodynamically relevant coronary stenosis}

CT-MPI revealed a territorial perfusion defect under adenosine stress (defined as a MBF under $75 \mathrm{ml} / 100 \mathrm{ml} / \mathrm{min}$ ) in 45 of 65 patients $(69.2 \%)$, whereas 20 patients had no territorial perfusion defect under adenosine stress (30.7\%); 33 (73.3\%) of the 45 patients having a territorial perfusion defect under adenosine stress, demonstrated a haemodynamically relevant coronary stenosis on FFR. Of the remaining 20 patients without any perfusion defect, 19 patients had no haemodynamically relevant coronary stenosis on FFR, one patient of these 20 without a perfusion defect under adenosine stress revealed a haemodynamically relevant coronary stenosis on FFR. Overall sensitivity, specificity, negative and PPV and DA of CT-MPI for the detection of a haemodynamically significant stenosis were 97, 65.6, 95.5, 74.4 and 81.5\%, respectively. As for CTA, these results persisted in a vessel-based analysis, and there was no difference in DA between the single coronary arteries (see table 2).

\section{Combination of $a \geq 50 \%$ stenosis on CTA and perfusion defect on CT-MPI for the detection of haemodynamically relevant coronary stenoses}

In this analysis, only patients with a stenosis $\geq 50 \%$ on CTA and a perfusion defect in the perfusion images were rated as having a haemodynamically significant stenosis. This means that patients with a stenosis $\geq 50 \%$ on CTA but without a perfusion defect on perfusion imaging, or inversely, with a perfusion defect on perfusion imaging but without stenosis $\geq 50 \%$ on CTA, were rated as having no haemodynamically significant stenoses.

Of the 65 patients investigated, 44 (67.9\%) had a stenosis $\geq 50 \%$ on CTA, and a territorial perfusion defect under adenosine stress, 21 patients (32.1\%) did not. Of the 44 patients having a stenosis $\geq 50 \%$ on CTA and a perfusion defect on CT-MPI, 33 (75\%) also had a haemodynamically relevant coronary stenosis on FFR while $11(25 \%)$ did not. From the 21 patients with a negative finding on CTA, 20 had no haemodynamically relevant coronary stenoses on FFR, while one patient showed a positive FFR measuring. Overall sensitivity, specificity, negative and PPV and DA of the combination of a $\geq 50 \%$ stenosis on CTA and perfusion defect on CT-MPI for the detection of a haemodynamically significant stenosis were 97, 68.8, 95.7, 76.1 and 83.1\%, respectively. Again, these results persisted without significant discord in a patient-based and vessel-based analysis. Again, no difference in DA between the single vessels was found (see table 2).

The combination of CTA and CT-MPI showed a very high sensitivity for the detection of a stenosis $\geq 50 \%$ as well as a very high NPV for the exclusion of a stenosis. While specificity, PPV 
Table 2 Sensitivity, specificity, negative predictive value (NPV), positive predictive value (PPV) and diagnostic accuracy (DA) with 95\% CI for CT angiography (CTA), CT-myocardial perfusion, and the combination of both for detecting haemodynamically relevant coronary artery stenoses defined by FFR

\begin{tabular}{|c|c|c|c|c|c|}
\hline & Sensitivity & Specificity & NPV & PPV & DA \\
\hline \multicolumn{6}{|l|}{ Overall $n=65$} \\
\hline CTA (\%) $(95 \% \mathrm{Cl})$ & $100(0.84$ to 1.0$)$ & $43.8(0.18$ to 0.65$)$ & $100(0.82$ to 1.0$)$ & $67.3(0.5$ to 0.9$)$ & 72 (0.54 to 0.86$)$ \\
\hline Perfusion (\%) $(95 \% \mathrm{Cl})$ & 97 (0.76 to 0.99$)$ & $65.6(0.51$ to 0.84$)$ & 95.5 (0.84 to 0.98$)$ & 74.4 (0.49 to 0.9$)$ & 81.5 (0.65 to 0.97$)$ \\
\hline CTA+perfusion (\%) (95\% CI) & $97(0.79$ to 1.0$)$ & $68.8(0.47$ to 0.79$)$ & 95.7 (0.76 to 0.99$)$ & 76.1 (0.56 to 0.92$)$ & 83.1 (0.7 to 0.96$)$ \\
\hline \multicolumn{6}{|l|}{ Vessel $n=195$} \\
\hline CTA (\%) $(95 \% \mathrm{Cl})$ & $97.5(0.78$ to 1$)$ & $53.9(0.26$ to 0.74$)$ & $98.8(0.71$ to 1.0$)$ & $36.6(0.13$ to 0.61$)$ & $63.1(0.38$ to 0.85$)$ \\
\hline Perfusion $(\%)(95 \% \mathrm{CI})$ & $95.1(0.83$ to 0.98$)$ & 74.0 (0.5 to 0.86$)$ & $98.3(0.82$ to 1.0$)$ & $49.3(0.17$ to 0.69$)$ & $78.2(0.52$ to 0.93$)$ \\
\hline CTA+perfusion (\%) $(95 \% \mathrm{Cl})$ & 95.1 (0.79 to 0.99$)$ & 74.7 (0.47 to 0.89$)$ & $98.2(0.84$ to 1.0$)$ & $50.0(0.26$ to 0.65$)$ & 79.0 (0.63 to 0.93$)$ \\
\hline \multicolumn{6}{|l|}{ LAD } \\
\hline CTA (\%) $(95 \%$ CI) & 93.8 (0.79 to 0.98$)$ & 55.1 (0.28 to 0.76$)$ & 96.4 (0.78 to 0.99$)$ & $40.5(0.14$ to 0.63$)$ & $64.6(0.49$ to 0.81$)$ \\
\hline Perfusion (\%) $(95 \% \mathrm{Cl})$ & 93.7 (0.81 to 0.99$)$ & $81.6(0.74$ to 0.97$)$ & $97.6(0.84$ to 1.0$)$ & $62.5(0.32$ to 0.79$)$ & $84.5(0.68$ to 0.97$)$ \\
\hline CTA+perfusion (\%) (95\% CI) & $93.8(0.82$ to 0.98$)$ & 79.6 (0.68 to 0.95$)$ & $97.5(0.83$ to 1.0$)$ & $60.0(0.3$ to 0.77$)$ & $83.1(0.72$ to 0.97$)$ \\
\hline \multicolumn{6}{|l|}{$\mathrm{RCX}$} \\
\hline CTA (\%) $(95 \%$ CI) & $100(0.82$ to 1.0$)$ & $52.8(0.24$ to 0.71$)$ & $100(0.79$ to 1.0$)$ & $32.4(0.11$ to 0.56$)$ & $61.5(0.29$ to 0.8$)$ \\
\hline Perfusion (\%) (95\% CI) & 91.7 (0.84 to 0.98$)$ & 69.8 (0.46 to 0.82$)$ & $97.3(0.84$ to 1.0$)$ & $40.7(0.14$ to 0.65$)$ & $73.8(0.48$ to 0.9$)$ \\
\hline CTA+perfusion (\%) (95\% CI) & 91.7 (0.84 to 1.0$)$ & 71.7 (0.55 to 0.87$)$ & $97.4(0.76$ to 1.0$)$ & $42.3(0.17$ to 0.63$)$ & $75.4(0.55$ to 0.91$)$ \\
\hline \multicolumn{6}{|l|}{ RCA } \\
\hline CTA (\%) & $100(0.83$ to 1.0$)$ & $53.8(0.28$ to 0.7$)$ & $100(0.79$ to 1.0$)$ & $35.1(0.12$ to 0.58$)$ & $63.1(0.47$ to 0.83$)$ \\
\hline Perfusion (\%) & $100(0.82$ to 1.0$)$ & 71.2 (0.53 to 0.84$)$ & $100(0.83$ to 1.0$)$ & $46.4(0.17$ to 0.62$)$ & 76.9 (0.58 to 0.91$)$ \\
\hline CTA+perfusion (\%) & 100 (0.83 to 1.0$)$ & 73.1 (0.46 to 0.87 ) & $100(0.84$ to 1.0$)$ & $48.1(0.17$ to 0.7$)$ & 78.4 (0.58 to 0.91$)$ \\
\hline
\end{tabular}

and DA for the detection of a haemodynamically significant stenosis by CTA alone, were very low, there was a significant increase in specificity, PPV and DA, for both CT-MPI alone and the combination of CTA and CT-MPI, for the detection of a heamodynamically relevant stenosis $(p=0.023)$.

\section{DISCUSSION}

CT has been demonstrated to be a very effective tool for the exclusion of significant coronary artery stenosis in patients without known CAD. ${ }^{14}{ }^{15}$ On the other hand, in patients with known $\mathrm{CAD}$, or in a cohort with a high prevalence of CAD, due to its low specificity and PPV, CT is not recommended. In patients with known CAD, as shown by the FAME-study ${ }^{1}$ and the COURAGE-study, ${ }^{2}$ it is crucial for the patient's survival to treat stenoses which are haemodynamically relevant. Therefore, an imaging modality providing information about the haemodynamic significance of coronary artery stenoses, in addition to the morphologic information, is required.

As previously reported by our group, we used a dynamic adenosine-stress myocardial perfusion protocol to perform myocardial perfusion scanning. ${ }^{7}$ This dynamic protocol permits derivation of a quantitative measure of myocardial perfusion similar to the established CT-based perfusion in brain imaging. ${ }^{16}$ Presently, it is not clear whether this added value of 'dynamic' imaging with MBF quantification, provides further clinical information over 'static' imaging (ie, a single set of images obtained during early myocardial perfusion). So far, reported data in the literature demonstrated similar findings regarding radiation exposure and contrast agent use. Also, the DA of these two different approaches is comparable. ${ }^{78}$

Our protocol included a first step CTA, with an average effective radiation exposure of $2.9 \pm 0.9 \mathrm{mSv}$, and in a second step, CT myocardial perfusion with a mean effective radiation exposure of $9.7 \pm 2.2 \mathrm{mSv}$. This also included the application of contrast agent twice.
By comparison with other modalities, such as SPECT- MPI and SP- MRI, CT-MPI achieved results in the diagnostic range of SPECT-MPI and SP-MRI. Still, it has to be taken into account that SPECT-MPI and SP-MRI evidence is based on thousands of published patients in numerous clinical trials. Further, the accuracy could deteriorate after distributing the method into different clinical populations and multicentre use.

Ho et al, ${ }^{17}$ comparing CT-MPI with SPECT-MPI, described a sensitivity of $95 \%$ and a specificity of $83 \%$ for the detection of haemodynamically relevant senosis using a 128 -slice scanner. George et al, ${ }^{18}$ investigating a patient collective similar to ours with suspected or known CAD, found a sensitivity of 100\% and a specificity of $81 \%$ using a 320 -slice scanner.

Similar results where reported by Feuchtner et $a l^{19}$ comparing SP-MPI to CT-MPI with a sensitivity of $96 \%$ and a specificity of $95 \%$ also in a collective of patients with suspected or known CAD and by Weininger et $a l^{20}$ with a sensitivity of $93 \%$ and a specificity of $99 \%$ in patients with acute chest pain.

The aim of this study was to evaluate the diagnostic benefit of CT-MPI in combination with CTA against CT-MPI alone in a larger study population with known (74.3\%) and suspected CAD (25.7\%). Invasive angiography revealed a stenosis above $50 \%$ in $55(84.6 \%)$ and a haemodynamically relevant stenosis in $33(50.8 \%)$ patients. Thus, a reasonable patient selection representing a population scheduled for invasive angiography can be assumed.

As described previously, we defined the cut-off point for MBF on the basis of maximisation of the area under the curve. We calculated a MBF of $75 \mathrm{ml} / 100 \mathrm{ml} / \mathrm{min}$ as an ideal cut-off point. However, this parameter may need further validation. Patients with an average MBF higher than this threshold level could be incorrectly diagnosed as having a non-haemodynamically significant stenosis.

CTA has proven to be a very sensitive tool in ruling out coronary artery stenosis in patients with a low to intermediate 
pretest likelihood for coronary artery stenosis. ${ }^{14} 15 \quad 21$ In this context, CTA has a very high sensitivity and NPV for ruling out significant coronary artery stenoses. These findings have also been consistently demonstrated in our series with a sensitivity of $100 \%$ and a NPV of $100 \%$ in ruling out haemodynamically relevant stenosis. There was no patient with an exclusion of coronary artery stenosis in CTA, who had a high-grade stenosis in coronary angiography or a stenosis defined as haemodynamically significant by FFR. If CTA already ruled out significant coronary artery stenoses, CT-MPI did not further improve DA. Therefore, we could have refrained from performing CT-MPI in seven patients in our study with no loss of information required for adequate clinical treatment, and with a consequent reduction of radiation exposure and contrast agent use. (figure 1)

In patients with CAD in CTA, Meijboom et $a l^{22}$ described a significant correlation between the quantitative assessment of coronary artery stenosis in CTA and angiographic findings. Still, the correlation with haemodynamic parameters determined by FFR was poor and had a sensitivity of approximately $50 \%$. Again, these findings were consistent in our series with a specificity of $43.8 \%$ and a PPV of $67.3 \%$, resulting in a DA of $72 \%$ for CTA alone in detecting haemodynamically relevant stenoses.
By contrast, CT-MPI had a significantly higher specificity $(65.6 \%)$ with a PPV of $74.4 \%$, resulting in a significant increase of DA up to $81.5 \%$ for the detection of a haemodynamically significant stenosis as defined by FFR. A comparable DA has been described in SPECT studies. Additionally, the mean effective radiation dose of $9.7 \pm 2.2 \mathrm{mSv}$ was comparable with nuclear techniques with the use of SPECT. ${ }^{23} 24$

The combination of CTA and CT-MPI demonstrated no significant further improvement in specificity (68.8\%), PPV (76.1\%) and DA (83.1\%) for the detection of haemodynamically significant coronary artery stenoses as defined by FFR in comparison to CT-MPI alone.

Based on these results, in patients with known CAD, or after stent implantation, CT-MPI alone might be sufficient, as the exclusion of haemodynamically relevant stenosis would not lead to a change in the treatment regime. Nevertheless, it might be reasonable to combine CTA and CT-MPI in patients without known CAD, but with an intermediate likelihood for CAD. Should CT-MPI reveal a perfusion defect under adenosine-stress, the patient might be suitable for coronary angiography and revascularisation. (figure 2) If there is no myocardial perfusion defect under adenosine-stress, but CTA reveals
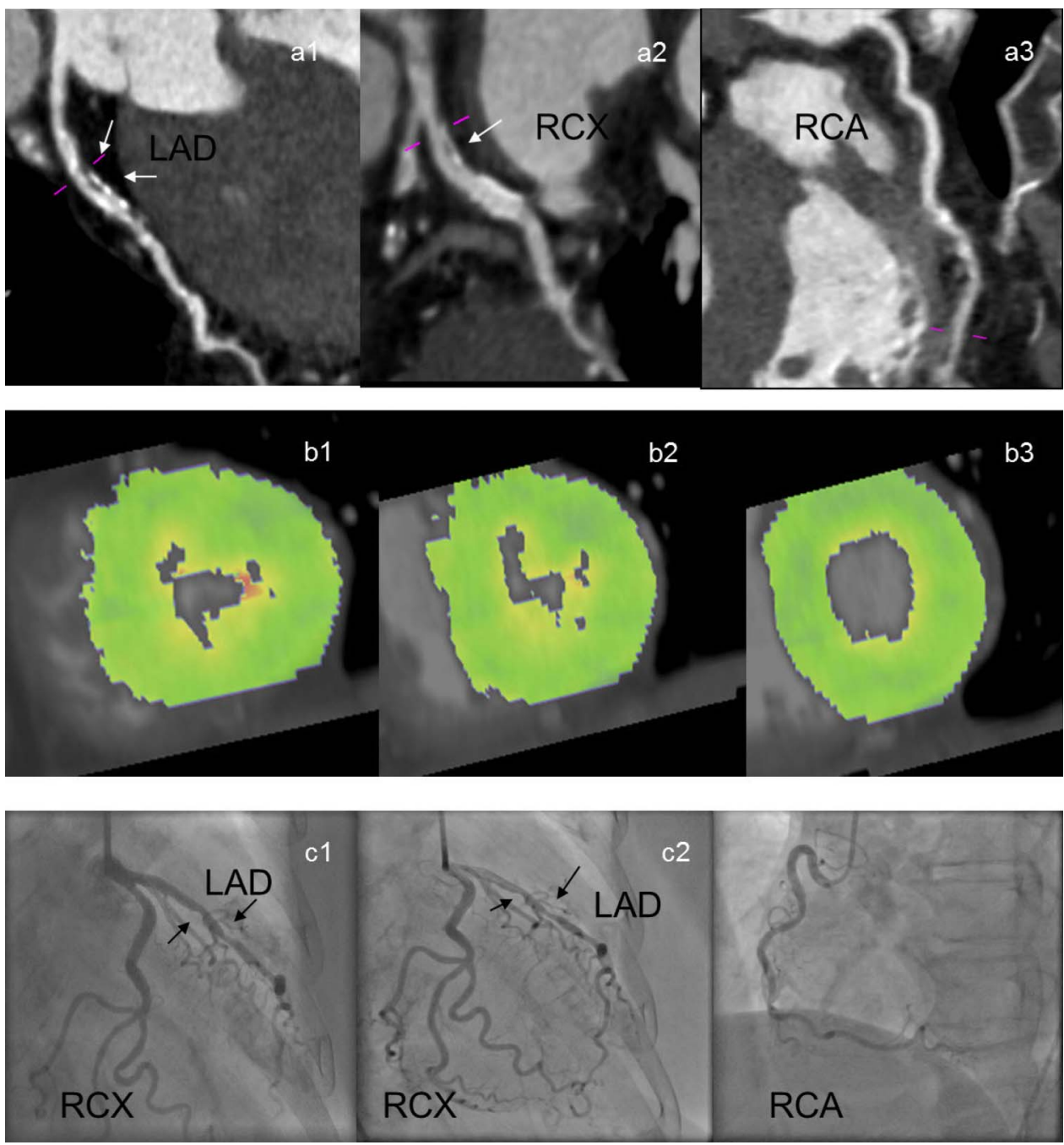

Figure 1 CTA reveals a mixed plaque in the proximal LAD with suspected stenosis (a1). The LCX shows a stent with a smaller mixed plaque proximal to the stent (a2). The RCA shows no plaque formation (a3). CT-MPI reveals no perfusion defect in the myocardium after adenosine stress (apical b1, mid b2, basal b3). MBF $>75 \mathrm{ml} / 100 \mathrm{ml} / \mathrm{min}$ (marked in green and yellow)) Coronary angiography reveals a tandem stenosis in the LAD (c1 and $\mathrm{c2}$ ). FFR measurement after adenosine stress reveals a haemodynamically not relevant tandem stenosis in the LAD with a FFR value of 0.83 . 

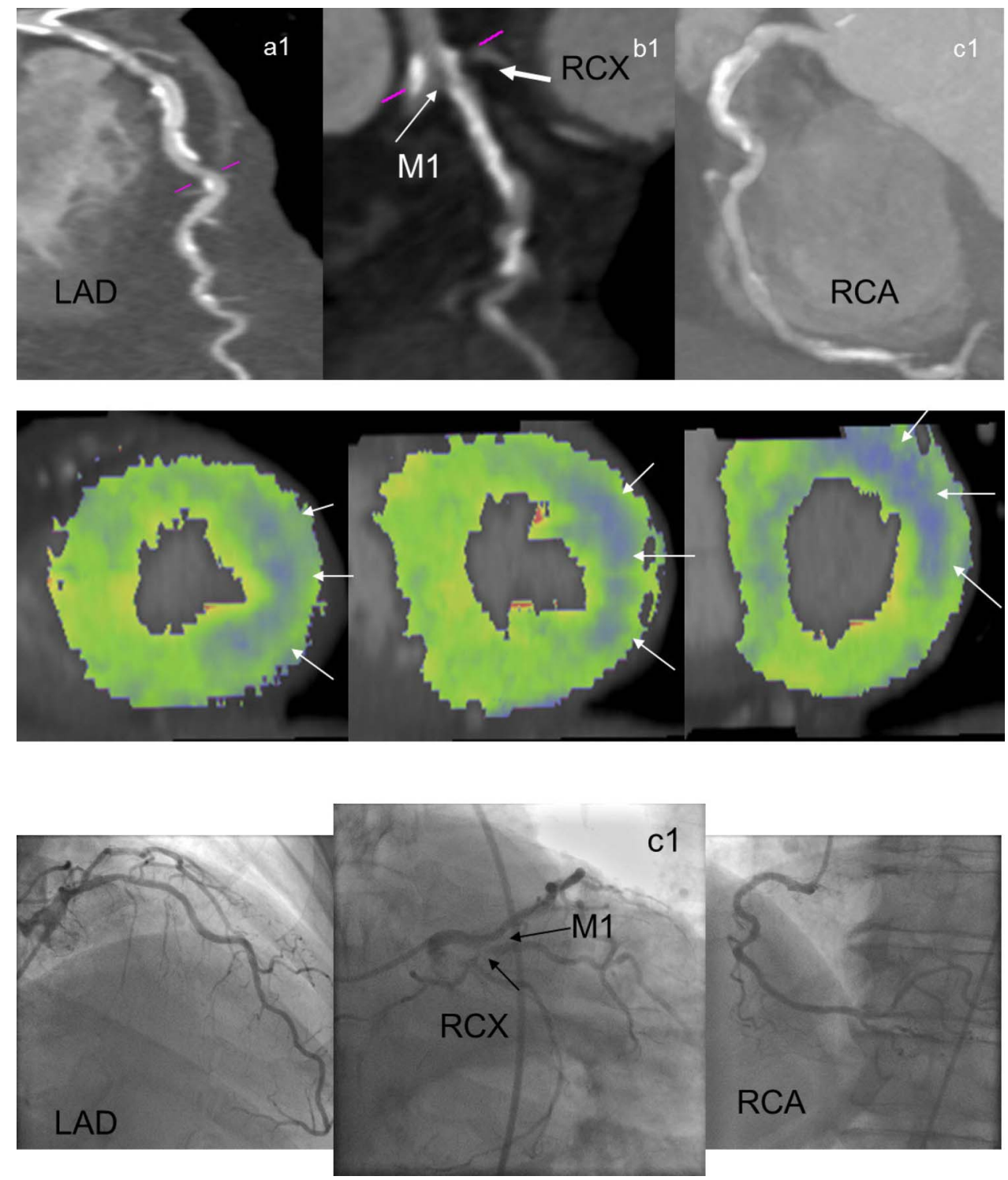

Figure 2 CTA shows many calcified plaques in the LAD (a1). The origin of the big first marginal branch (M1), as well as the origin of the smaller LCX, show a significant stenosis (a2). The RCA shows a calcified plaque in the proximal part (a3). CT-MPI reveals a significant perfusion defect under adenosine stress (MBF $<75 \mathrm{ml} / 100 \mathrm{ml} / \mathrm{min}$ ) marked blue/grey in the LCX territories. Of note, as the LCX is a large vessel with two branches running towards the RCA territories. Thus, a part of the basal septal segment coloured in blue/grey indicates a perfusion defect in the borderline segments (apical, mid, basal). Coronary angiography shows a high-grade stenosis on the origin of the first marginal branch (M1) as well as of the origin of the LCX with a FFR of 0.69 and 0.72 , respectively. (c1) There was no coronary artery stenosis in the LAD or RCA.

coronary artery plaque formation, or a haemodynamically nonsignificant coronary artery stenosis, a preventive medical treatment (eg, aspirin, statins) might be favourable (figure 3). In this context, CT is currently the sole available imaging tool which offers the unique possibility to anatomically visualise the coronary arteries and their pathological changes, and also enables the determination the haemodynamic significance of any detected stenosis.

\section{Limitations}

Our study has several limitations. First, selection bias may be present because patients included presented with typical or atypical chest pain, and had an intermediate to high risk for the presence of coronary artery stenosis. Further, the PPV and NPV are dependent on the prevalence of disease. Thus, our results are limited to populations with the same prevalence as in our series. Nevertheless, this is the typical patient population to be referred for a stress test. Still, patients with previous myocardial infarction were excluded.

Second, to achieve optimal results, the patients need adequate preparation and should be able to hold their breath for almost $30 \mathrm{~s}$, otherwise, breathing artefacts or suboptimal vasodilatory stress influence image quality and results. This limits the perfusion study to a patient population in a relatively good clinical condition.

Third, it is important to point out, that the MBF calculated in CT MPI cannot be considered to be the absolute blood flow. At its best, it is a semiquantitative analysis, which allows to compare different myocardial regions. This might explain the values beyond the physiological range.

Fourth, further work is needed to improve our scanning protocol to reduce radiation exposure. Finally, this is a single-centre study, and further research is needed to confirm our findings. 

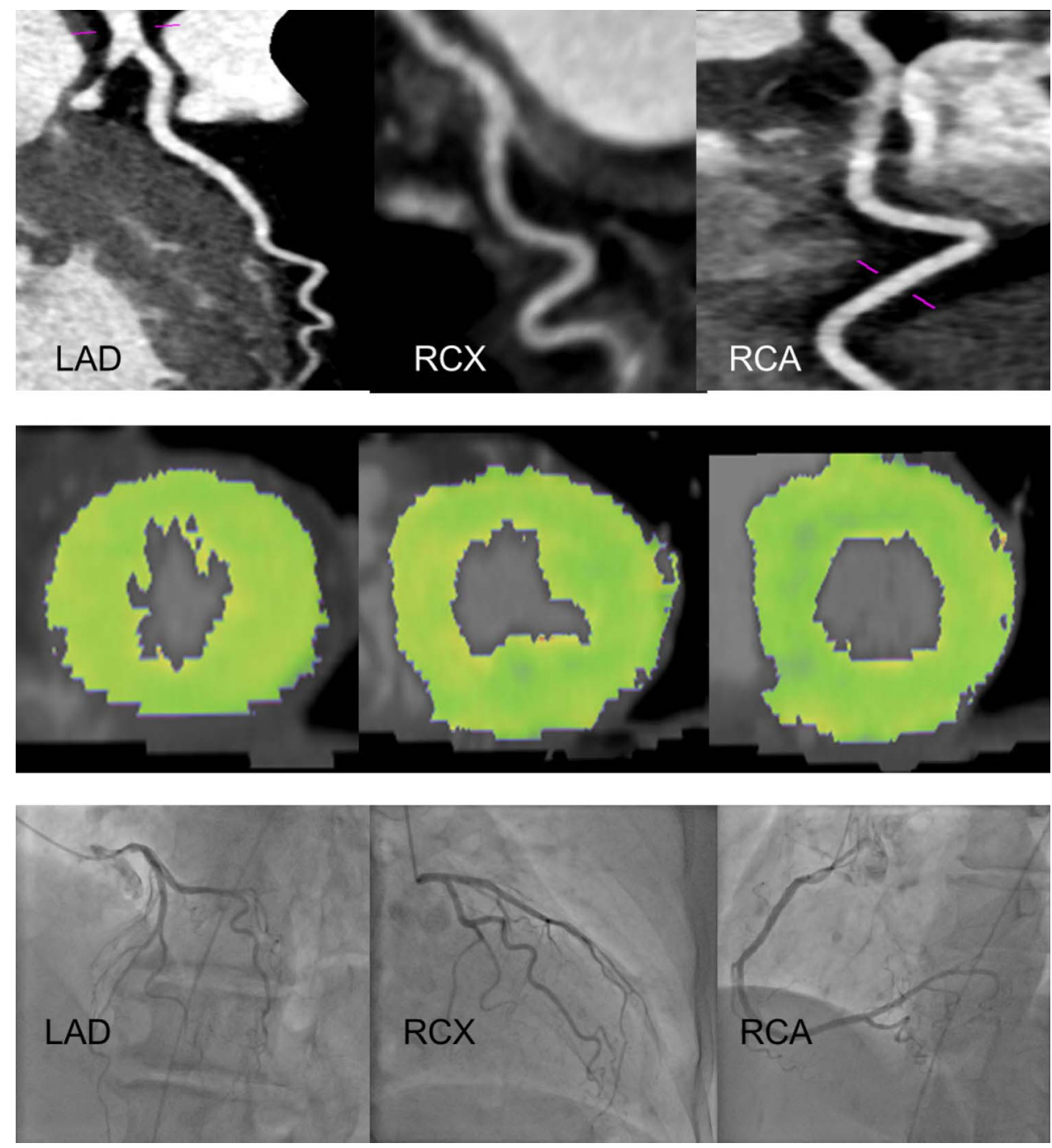

Figure 3 CTA shows no plaque formation in the coronary vessels. CT-MPI shows no perfusion defect under adenosine stress. MBF $>75 \mathrm{ml} / 100 \mathrm{ml} /$ min (marked with green/yellow) (apical, mid, basal). Coronary angiography shows no coronary stenosis.

\section{CONCLUSION}

Our data suggest that CT-MPI permits detection of haemodynamic relevant coronary artery stenoses with a moderate DA. CT may, therefore, allow the simultaneous assessment of both coronary morphology and function.

Contributors MG: initiation of the study (concept), data collection and analysis, manuscript writing. FB: concept of the study, CT image analysis. FvZ: patient recruitment, $\mathrm{CT}$ image analysis. JT: patient recruitment, coronary angiography analysis. FS and RPM: CT image analysis. MD: manuscript review, language correction.JS: patient recruitment. CB: manuscript review. KN: concept of the study, $\mathrm{CT}$ image analysis. AB: concept of the study, CT image analysis, manuscript writing and review.

\section{Competing interests None.}

Patient consent Obtained.

Ethics approval The study was approved by the institutional review board and the Federal Radiation Safety Council (Bundesamt für Strahlenschutz).

Provenance and peer review Not commissioned; externally peer reviewed.

\section{REFERENCES}

1 Pijls NH, Fearon WF, Tonino PA, et al. Fractional flow reserve versus angiography for guiding percutaneous coronary intervention in patients with multivessel coronary artery disease: 2-year follow-up of the FAME (Fractional Flow Reserve Versus Angiography for Multivessel Evaluation) study. J Am Coll Cardiol 2010;56:177-84.

2 Boden WE, O'Rourke RA, Teo KK, et al. Optimal medical therapy with or without $\mathrm{PCl}$ for stable coronary disease. N Engl J Med 2007;356:1503-16.
3 Nieman K, Shapiro MD, Ferencik M, et al. Reperfused myocardial infarction: contrastenhanced 64-Section CT in comparison to MR imaging. Radiology 2008;247:49-56.

4 George RT, Silva C, Cordeiro MA, et al. Multidetector computed tomography myocardial perfusion imaging during adenosine stress. J Am Coll Cardiol 2006:48:153-60.

5 Blankstein R, Shturman LD, Rogers IS, et al. Adenosine-induced stress myocardial perfusion imaging using dual-source cardiac computed tomography. J Am Coll Cardiol 2009;54:1072-84

6 George RT, Arbab-Zadeh A, Miller JM, et al. Sine stress 64- and 256-row detector computed tomography angiography and perfusion imaging: a pilot study evaluating the transmural extent of perfusion abnormalities to predict atherosclerosis causing myocardial ischemia. Circ Cardiovasc Imaging 2009:2:174-82.

7 Bamberg F, Becker A, Schwarz F, et al. Detection of hemodynamically significant coronary artery stenosis: incremental diagnostic value of dynamic CT-based myocardial perfusion imaging. Radiology 2011;260:689-98.

8 Ko BS, Cameron JD, Meredith IT, et al. Computed tomography stress myocardial perfusion imaging in patients considered for revascularization: a comparison with fractional flow reserve. Eur Heart J 2012;33:67-77.

9 Cerqueira MD, Weissman NJ, Dilsizian V, et al. Standardized myocardial segmentation and nomenclature for tomographic imaging of the heart: a statement for healthcare professionals from the Cardiac Imaging Committee of the Council on Clinical Cardiology of the American Heart Association. Circulation 2002;105:539-42.

10 Bruder $\mathrm{H}$, Raupach R, Klotz E, et al. Spatio-temporal filtration of dynamic CT data using diffusion filters. In: Samei E, Hsieh J. Proceedings of SOIE: medical imaging 2009-physics of medical imaging. Vol 7258. Bellingham, Wash: SPIE-the International Society for Optical Engineering, 2009:725857.

11 Bamberg F, Klotz E, Flohr T, et al. Dynamic myocardial stress perfusion imaging using fast dual-source CT with alternating table positions: initial experience. Eur Radiol 2010;20:1168-73. 
12 Hanley JA, McNeil BJ. The meaning and use of the area under a receiver operating characteristic (ROC) curve. Radiology 1982;143:29-36.

13 DeLong ER, DeLong DM, Clarke-Pearson DL. Comparing the areas under two or more correlated receiver operating characteristic curves: a nonparametric approach. Biometrics 1988:44:837-45.

14 Taylor AJ, Cerqueira M, Hodgson JM, et al. ACCF/SCCT/ACR/AHA/ASE/ASNC/ NASCI/SCAI/SCMR 2010. Appropriate Use Criteria for Cardiac Computed Tomography. A Report of the American College of Cardiology Foundation Appropriate Use Criteria Task Force, the Society of Cardiovascular Computed Tomography, the American College of Radiology, the American Heart Association, the American Society of Echocardiography, the American Society of Nuclear Cardiology, the North American Society for Cardiovascular Imaging, the Society for Cardiovascular Angiography and Interventions, and the Society for Cardiovascular Magnetic Resonance. Circulation 2010;122: e525-55.

15 Budoff MJ, Achenbach S, Blumenthal RS, et al. American Heart Association Committee on Cardiovascular Imaging and Intervention; American Heart Association Council on Cardiovascular Radiology and Intervention; American Heart Association Committee on Cardiac Imaging, Council on Clinical Cardiology. Assessment of coronary artery disease by cardiac computed tomography: a scientific statement from the American Heart Association Committee on Cardiovascular Imaging and Intervention, Council on Cardiovascular Radiology and Intervention, and Committee on Cardiac Imaging, Council on Clinical Cardiology. Circulation 2006;114:1761-91.

16 Muizelaar JP, Fatouros PP, Schröder ML. A new method for quantitative regional cerebral blood volume measurements using computed tomography. Stroke 1997;28:1998-2005.
17 Ho KT, Chua KC, Klotz E, et al. Stress and rest dynamic myocardial perfusion imaging by evaluation of complete time-attenuation curves with dual-source CT. JACC Cardiovasc Imaging 2010;3:811-20.

18 George RT, Arbab-Zadeh A, Miller JM, et al. Computed tomography myocardial perfusion imaging with 320-row detector computed tomography accurately detects myocardial ischemia in patients with obstructive coronary artery disease. Circ Cardiovasc Imaging 2012;5:333-40.

19 Feuchtner G, Goetti R, Plass A, et al. Adenosine stress high-pitch 128-slice dual-source myocardial computed tomography perfusion for imaging of reversible myocardial ischemia: comparison with magnetic resonance imaging. Circ Cardiovasc Imaging 2011;4:540-9.

20 Weininger M, Schoepf UJ, Ramachandra A, et al. Adenosine-stress dynamic real-time myocardial perfusion $\mathrm{CT}$ and adenosine-stress first-pass dual-energy myocardial perfusion $\mathrm{CT}$ for the assessment of acute chest pain: initial results. Eur J Radiol 2012;81:3703-3710.

21 Leber AW, Johnson T, Becker A, et al. Diagnostic accuracy of dual-source multi-slice CT-coronary angiography in patients with an intermediate pretest likelihood for coronary artery disease. Eur Heart J 2007;28:2354-60.

22 Meijboom WB, Van Mieghem CA, van Pelt N, et al. Comprehensive assessment of coronary artery stenoses: computed tomography coronary angiography versus conventional coronary angiography and correlation with fractional flow reserve in patients with stable angina. Am Coll Cardiol 2008;52:636-43.

23 Cerqueira MD, Allman KC, Ficaro EP, et al. Recommendations for reducing radiation exposure in myocardial perfusion imaging. J NuCl Cardiol 2010;17:709-18.

24 Bateman TM, Heller GV, McGhie Al, et al. Diagnostic accuracy of rest/stress ECG-gated Rb-82 myocardial perfusion PET: comparison with ECG-gated Tc-99m sestamibi SPECT. J Nucl Cardiol 2006;13:24-33. 
CT stress perfusion imaging for detection of haemodynamically relevant coronary stenosis as defined by FFR

Martin Greif, Franz von Ziegler, Fabian Bamberg, Janine Tittus, Florian Schwarz, Melvin D'Anastasi, Roy P Marcus, Jan Schenzle, Christoph Becker, Konstantin Nikolaou and Alexander Becker

Heart 2013 99: 1004-1011 originally published online May 14, 2013 doi: 10.1136/heartjnl-2013-303794

Updated information and services can be found at:

http://heart.bmj.com/content/99/14/1004

\section{These include:}

References This article cites 23 articles, 9 of which you can access for free at: http://heart.bmj.com/content/99/14/1004\#BIBL

Email alerting Receive free email alerts when new articles cite this article. Sign up in the service box at the top right corner of the online article.

Topic Articles on similar topics can be found in the following collections Collections

\section{Notes}

To request permissions go to:

http://group.bmj.com/group/rights-licensing/permissions

To order reprints go to:

http://journals.bmj.com/cgi/reprintform

To subscribe to BMJ go to:

http://group.bmj.com/subscribe/ 